\title{
Synthesis of Dexamethasone
}

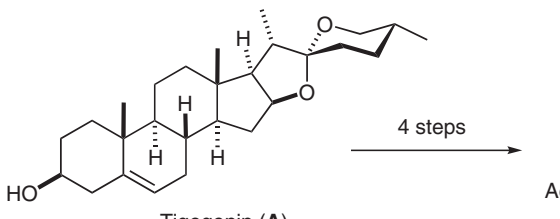

Tigogenin (A)

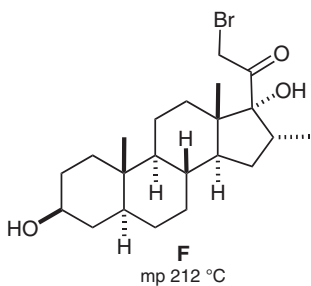

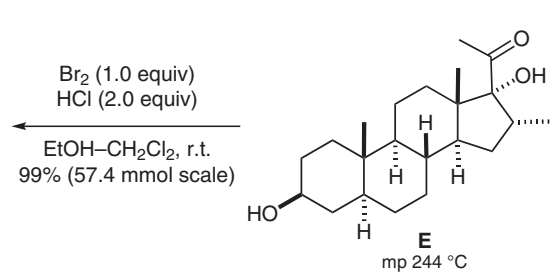

${ }^{*}$ [ ] denotes a product used in the
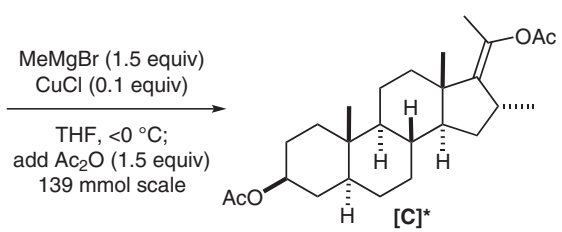

$\mathrm{MeCO}_{3} \mathrm{H}$ (3.8 equiv) 1,2-DCE $\mathrm{NaOAc}(25 \mathrm{~g})$

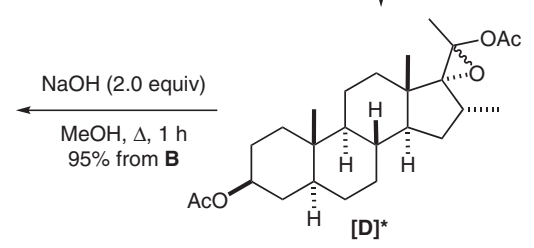

Synthesis of Natural

Products and

Potential Drugs

\section{Key words}

dexamethasone

corticosteroid

tigogenin

COVID-19

conjugate addition
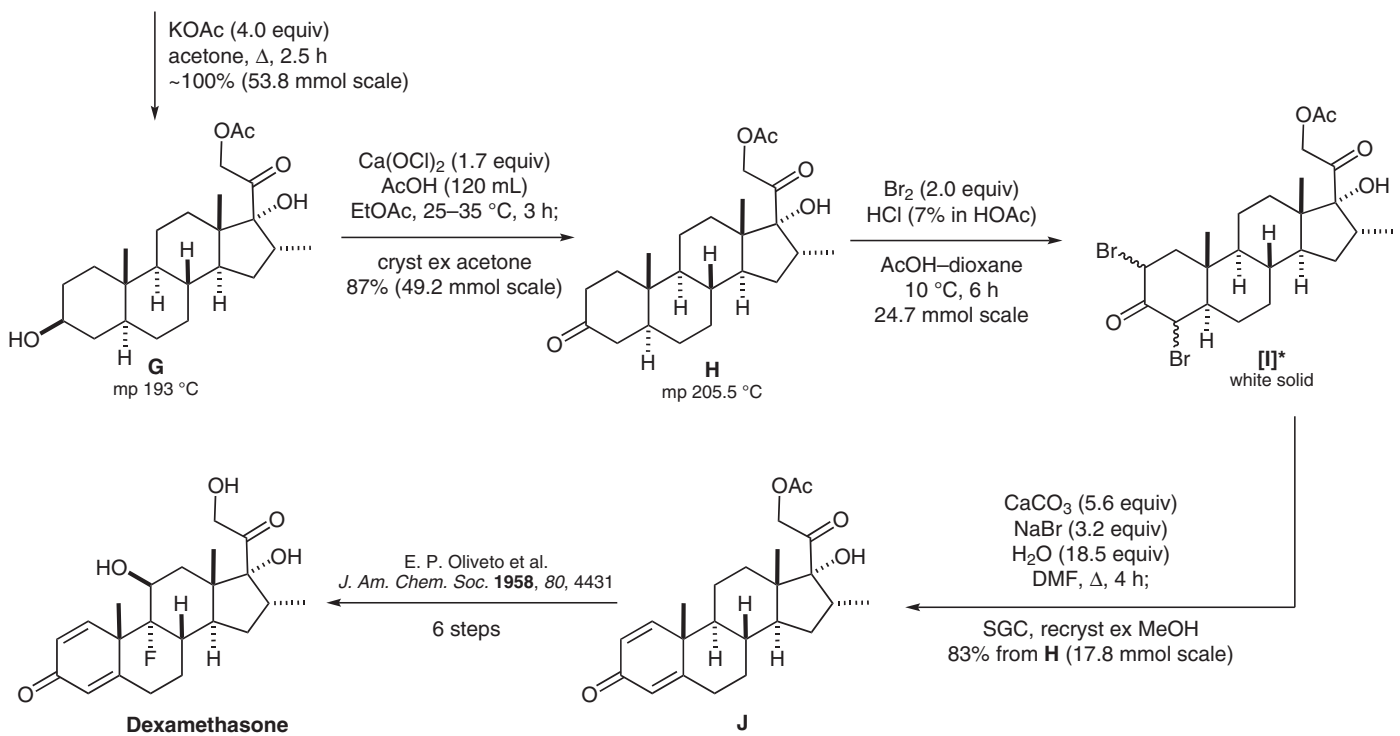

Significance: Dexamethasone is a corticosteroid that was first synthesized in 1957 and was approved in 1961 for the treatment of a wide range of inflammatory disorders. It is on the WHO List of Essential Medicines. In June 2020, dexamethasone was given preliminary approval for the treatment of COVID-19 because it improved survival rates of hospitalized patients receiving oxygen or on a ventilator. In 1997, Furukawa and co-workers reported a synthesis of dexamethasone from tigogenin.
Comment: For the introduction of the $17 \alpha$-hydroxy-16 $\alpha$-methyl moiety $(\mathbf{B} \rightarrow \mathbf{E}$ ) the key epoxidation reaction was accomplished with peracetic acid in a buffer solution of sodium acetate and acetic acid (95\% overall yield). Then the introduction of the 1,4-diene in the A-ring $(\mathbf{H} \rightarrow \mathbf{J}, 83 \%$ yield) was greatly improved by bromination-dehydrobromination, in which dehydrobromination proceeded smoothly in a mixture of DMF and $6 \%$ of water $(83 \%$ yield). 